\title{
Estimación de la Eficiencia de una Hélice Operando en Régimen Compresible Subsónico
}

\section{Estimation of the Efficiency of a Propeller Operating in Subsonic Compressible} Regime

DOI: $10.46932 / \mathrm{sfjdv2n4-007}$

Received in: March 1st, 2021

Accepted in: May 30th, 2021

Tiburcio Fernández Roque

Doctor en Ciencias en Ingeniería Mecánica por la Escuela Superior de Ingeniería Mecánica y Eléctrica (ESIME) del Instituto Politécnico Nacional (I.P.N.).

Avenida Ticoman número 600, colonia San José Ticomán, alcaldía Gustavo A. Madero, C.P. 07340, Ciudad de México, México.

E-mail: tfroque@yahoo.com.mx

\section{José Arturo Correa Arredondo}

Maestro en Ingeniería Aeronáutica por la Escuela Superior de Ingeniería Mecánica y Eléctrica (ESIME) del Instituto Politécnico Nacional (I.P.N.).

Avenida Ticoman número 600, colonia San José Ticomán, alcaldía Gustavo A. Madero, C.P. 07340, Ciudad de México, México.

E-mail: josearturocorrea@yahoo.com.mx

\section{José Félix Vázquez Flores}

Doctor en Ciencias en Ingeniería Mecánica por la Escuela Superior de Ingeniería Mecánica y Eléctrica (ESIME) del Instituto Politécnico Nacional (I.P.N.).

Avenida Ticoman número 600, colonia San José Ticomán, alcaldía Gustavo A. Madero, C.P. 07340,

Ciudad de México, México.

E-mail: bemol5808@gmail.com

\section{Jorge Sandoval Lezama}

Maestría en Sistemas de Control. Instituto Politécnico Nacional, Escuela Superior de Ingeniería Mecánica y Eléctrica Unidad Ticoman.

Avenida Ticoman número 600, colonia San José Ticomán, alcaldía Gustavo A. Madero, C.P. 07340, Ciudad de México, México.

E-mail: jslezama09@yahoo.com.mx

\section{Alejandro Mejía Carmona}

Ingeniero en Aeronáutica, Maestro en Ciencias de la Administración con especialidad en Desarrollo de la Educación por el Instituto Politécnico Nacional.

Avenida Ticoman número 600, colonia San José Ticomán, alcaldía Gustavo A. Madero, C.P. 07340,

Ciudad de México, México.

E-mail: almejia@ipn.mx 


\title{
RESUMEN
}

La información experimental disponible para hélices no es útil cuando el número de Mach de la punta de la pala es mayor a 0.3. Con el fin de verificar esta aseveración, se propuso un caso de estudio para una hélice Navy 5868-9 con perfil aerodinámico Clark-Y de 4 palas operando a un número de Mach de avance de 0.59 y un número de Mach en la punta de la pala de 0.95. La eficiencia experimental de la hélice (obtenida de gráficas) se compara con la eficiencia obtenida empleando la teoría combinada y con la eficiencia obtenida al corregir la teoría combinada por efectos de compresibilidad con la metodología propuesta en este trabajo. Se concluye que la información experimental disponible para hélices no es adecuada cuando el número de Mach en la punta de la pala es mayor que el Mach crítico, siendo más conveniente el resultado teórico corregido por compresibilidad.

Palabras clave: Hélice; Número de Mach; Teoría combinada elemento de pala-momentum.

\begin{abstract}
The experimental information available for propellers is not useful when the Mach number of the tip of the blade is greater than 0.3. In order to verify this assertion, a case study was proposed for a Navy propeller 5868-9 with a 4-blade Clark-Y airfoil section operating at an advance Mach number of 0.59 and a Mach number at the tip of the blade of 0.95 . The experimental efficiency of the propeller (obtained from graphs) is compared with the efficiency obtained using the combined theory and with the efficiency obtained by correcting the combined theory for compressibility effects with the methodology proposed in this paper. It is concluded that the experimental information available for propellers is not suitable when the Mach number at the tip of the blade is greater than the critical Mach, being more convenient the theoretical result corrected by compressibility.
\end{abstract}

Keywords: Propeller; Mach number; Combined theory blade element-momentum.

\section{INTRODUCCIÓN}

El rendimiento de una hélice es afectado favorablemente cuando el número de Mach en la punta de la pala es mayor a 0.3 y menor al número de Mach crítico $\left(0.65<M_{c r}<0.85\right.$, dependiendo de la geometría y del ángulo de ataque del perfil aerodinámico de cada elemento de pala) y en forma adversa cuando es mayor al número de Mach crítico, ya que la eficiencia de la hélice disminuye considerablemente. En la referencia [1] se indica que "el rendimiento de una hélice en el rango de velocidades de avance y de revoluciones de la hélice empleadas en el despegue de una aeronave es considerablemente afectada por la velocidad de la punta de la pala y que, para poder aplicar las correcciones necesarias cuando se utilizan los presentes datos, es necesario conocer la velocidad de la punta de pala de las pruebas realizadas para obtener dichos datos". Lo anterior también es cierto cuando la aeronave vuela en crucero a números de Mach mayores a 0.3 sobre todo si la hélice es de diámetro grande y gira a altas revoluciones. Sin embargo, la referencia [1] no indica cuales son las "correcciones necesarias". La Figura 1 es una gráfica obtenida de la referencia [1] que proporciona la eficiencia de una hélice Navy 5868-9 de 4 palas que emplea un perfil Clark Y, en función de la relación de avance de la hélice, $J=V / n D$, en donde $V$ es la velocidad de la aeronave, $n$ son las revoluciones por segundo de la 
hélice y $D$ es el diámetro de la hélice. Aunque en la referencia [1] no se indica cual es el número de Mach en la punta de la pala de la hélice, se estimó, con los datos que en dicha referencia se presentan, que dicho número es de 0.24 cuando $J=2.5$, es decir, la hélice probada operaba en un régimen incompresible.

Figura 1. Curvas de eficiencia para una hélice Navy 5868-9, perfil Clark Y, de cuatro palas. Tomada de [1].

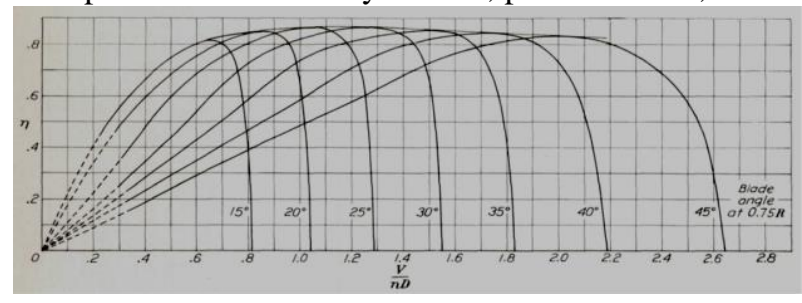

La referencia [2] proporciona la ecuación (1), la cual se emplea para corregir la eficiencia de una hélice, $\eta$, en función de su eficiencia nominal, $\eta_{\text {nominal }}$, la cual se puede obtener de las gráficas de la referencia [1], del número de Mach en la punta de la pala, $M_{t i p}$, y del número de Mach crítico, $M_{c r}$ :

$$
\eta=\eta_{\text {nominal }}-\frac{15}{100}\left(\frac{M_{t i p}-M_{c r}}{0.1}\right)
$$

Sin embargo, de los cálculos realizados para el caso de estudio aquí propuesto, se ha determinado que la ecuación (1) arroja eficiencias mayores a las calculadas con la metodología que aquí se propone. Por lo anterior, el objetivo de este trabajo es mostrar la metodología desarrollada para estimar la eficiencia de una hélice que opera en régimen compresible subsónico y compararla con los obtenidos con la Figura 1 y con la ecuación (1).

\section{METODOLOGÍA PROPUESTA}

La metodología que aquí se propone para la estimación de la eficiencia de una hélice se basa en la teoría combinada para una hélice, la cual combina la teoría del elemento de pala con la teoría del Momentum o teoría de Rankine-Froude. Los valores de los coeficientes de sustentación y de arrastre obtenidos con esta teoría, de los elementos de pala que requieran la corrección por número de Mach, se realiza empleando la información experimental disponible en las referencias consultadas. Una vez corregidos, los coeficientes aerodinámicos se utilizan para recalcular las velocidades inducidas axial y tangencial de los elementos de pala que lo requieran. Con las velocidades inducidas corregidas se calculan nuevamente los coeficientes de tracción y de potencia de cada elemento de pala y finalmente se calculan los coeficientes de tracción y de potencia de la hélice y la eficiencia de esta. En lo que sigue se detalla lo antes expuesto. 


\subsection{CÁLCULO DE UNA HÉLICE EMPLEANDO LA TEORÍA COMBINADA}

Empleando la teoría combinada podemos determinar las velocidades inducidas axial y tangencial, los coeficientes de sustentación y de arrastre y las contribuciones al coeficiente de tracción y al coeficiente de potencia de cada elemento de pala y con esto determinar el coeficiente de tracción, de potencia y la eficiencia de una hélice operando en régimen incompresible.

Para el cálculo de las variables antes indicadas se requiere conocer la geometría de la pala de la hélice, es decir:

1.- La distribución de cuerda con respecto al diámetro de la hélice, $c$.

2.- La distribución de espesores relativos de los elementos de pala, $t / c$.

3.- La distribución del ángulo de paso geométrico, $\beta$, a lo largo de la pala, con respecto a la cuerda geométrica de cada elemento de pala.

4.- Número de palas de la hélice, $B$.

También se debe de conocer las características aerodinámicas de cada uno de los elementos de pala, es decir:

5.- El ángulo de sustentación nula, $\alpha_{L=0}$.

6.- La pendiente de la parte recta de la curva de sustentación, $a_{0}$.

7.- El coeficiente de arrastre para sustentación nula, $C_{d 0}$.

8.- La constante para calcular el coeficiente de arrastre del elemento de pala con sustentación diferente de cero, $k$, el cual se considera que es el mismo para todos los elementos de pala.

Adicionalmente se debe conocer la relación de avance de la hélice, $J$, y establecer el número de elementos de pala en los que se divide la pala.

Puesto que el desarrollo de la teoría combinada para el cálculo de una hélice se encuentra en diversas referencias y libros de texto relacionados con hélices y aerogeneradores, aquí únicamente se indican las ecuaciones a utilizar y el procedimiento a seguir para el cálculo de la hélice. Las ecuaciones fueron tomadas de la referencia [3]. Las variables involucradas se pueden ver en la Figura 2. 
Figura 2.- Variables en un elemento de pala con base a la teoría combinada. Adaptada de [3].

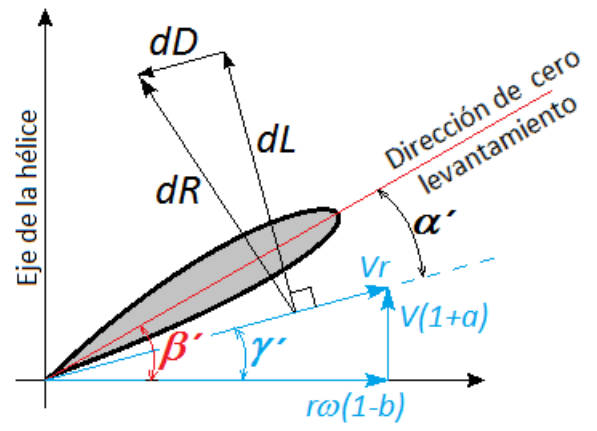

1.- El cálculo se inicia suponiendo un valor de coeficiente de levantamiento $C_{l}$, para un determinado elemento de pala.

2.- Se calcula el ángulo de ataque absoluto, $\alpha^{\prime}=\frac{c_{l}}{a_{0}}$.

3.- Se calcula el ángulo de paso del elemento de pala con respecto a la cuerda de sustentación nula (cuerda aerodinámica), $\beta^{\prime}=\beta-\alpha_{L=0}$.

4.- Se calcula el ángulo formado por la velocidad resultante corregida por las velocidades inducidas axial y tangencial con respecto al plano de rotación, $\gamma^{\prime}=\beta^{\prime}-\alpha^{\prime}$.

5.- Se calcula el coeficiente de arrastre del elemento de pala, $C_{d}=C_{d 0}+k C_{l}^{2}$.

6.- Se calcula la velocidad inducida axial adimensional, $a=\left[\frac{4 \operatorname{sen}^{2}\left(\gamma^{\prime}\right)}{s\left(C_{l} \cos \left(\gamma^{\prime}\right)-C_{d} \operatorname{sen}\left(\gamma^{\prime}\right)\right)}-1\right]^{-1}$. En esta ecuación, $s$ es la solidez de los $B$ elementos de pala que se encuentran en la misma estación $r$ y se calcula con la siguiente ecuación: $s=\frac{B c}{\pi r}$.

7.- Se calcula la velocidad inducida tangencial adimensional, $b=\left[\frac{4 \operatorname{sen}\left(\gamma^{\prime}\right) \cos \left(\gamma^{\prime}\right)}{s\left(C_{l} \operatorname{sen}\left(\gamma^{\prime}\right)+C_{d} \cos \left(\gamma^{\prime}\right)\right)}+1\right]^{-1}$.

8.- Se calcula una nueva $\gamma^{\prime}, \gamma_{n}^{\prime}=\operatorname{atan}\left(\frac{J(1+a)}{\pi r(1-b)}\right)$. 
9.- Se compara la $\gamma^{\prime}$ calculada en el paso 4 con la calculada en el paso $8, \gamma_{n}^{\prime}$, si son diferentes se cambia el $C_{l}$ supuesto en el paso 1 y se repiten los pasos 2 al 7 . El proceso se repite tantas veces como sea necesario hasta que la última $\gamma^{\prime}$ sea igual o aproximadamente igual a la de la serie anterior. Cuando son iguales las dos $\gamma^{\prime}$ termina el cálculo para el elemento de pala en cuestión, obteniéndose los valores de $a, b, \gamma^{\prime}, \alpha^{\prime}, C_{l} y C_{d}$. Esto se hace para cada elemento de pala en los que se haya dividido la pala de la hélice.

10.- Se calcula el diferencial del coeficiente de tracción con respecto al radio adimensional, $\frac{d C_{T}}{d r}=$ $\pi a J^{2} r(1+a)$, y el diferencial de potencia con respecto al radio adimensional, $\frac{d C_{p}}{d r}=\pi^{3} b J r^{3}(1+a)$, de cada elemento de pala.

11.- Se calcula el coeficiente de tracción, $C_{T}$, y el coeficiente de potencia, $C_{p}$, de la hélice integrando la distribución de $\frac{d C_{T}}{d r}$ y de $\frac{d C_{p}}{d r}$ a lo largo del radio adimensional de la hélice, $r$. En el caso analizado esto se hizo empleando la función trapz del MatLab.

12.- Se calcula el rendimiento de la hélice, $\eta=\frac{J C_{T}}{C_{p}}$

\subsection{CORRECCIÓN POR NÚMERO DE MACH}

Los coeficientes de tracción y de potencia y el rendimiento de la hélice calculados en el punto 2, se considera que son para un flujo incompresible ya que los datos aerodinámicos utilizados corresponden a un número de Mach menor a 0.3, por lo tanto, puesto que algunos elementos de pala de la hélice que se desea analizar pueden operar a un número de Mach mayor a 0.3, los efectos de compresibilidad son importantes y es necesario efectuar la corrección por número de Mach. Esta corrección es empírica utilizando información experimental disponible para la variación de los coeficientes aerodinámico con el número de Mach. Este procedimiento se considera que es la mayor aportación de este trabajo ya que en la búsqueda de información realizada no se encontró algo similar a lo que aquí se propone.

La corrección que se hace por el número de Mach al cual opera el elemento de pala se aplicó al coeficiente de levantamiento y al coeficiente de arrastre del elemento de pala tomando en cuenta el ángulo de ataque absoluto del mismo, $\alpha^{\prime}$. Para esto se requiere calcular primeramente el número de Mach de cada elemento de pala. Esto se hace con el siguiente procedimiento. 
1.- Se calcula la velocidad resultante adimensional del elemento de pala, $J_{R e}=$ $\sqrt[2]{J^{2}(1+a)^{2}+[\pi r(1-b)]^{2}}$

2.- Se calcula la velocidad resultante adimensional de la punta de la pala de la hélice, $J_{R p}=\sqrt[2]{J^{2}+\pi^{2}}$.

3.- Si se establece el número de Mach para la punta de pala, $M_{p}$, se calcula la velocidad del sonido adimensional, $J_{V s}=J_{R p} / M_{p}$.

4.- Se calcula el número de Mach del elemento de pala, $M_{e}=\frac{J_{R e}}{J_{V s}}$.

Una vez determinado el número de Mach al que opera cada elemento de pala se corrige el coeficiente de levantamiento y el coeficiente de arrastre calculados con la teoría combinada, de los elementos de pala que lo requieran, es decir, los que tengan un número de Mach mayor a 0.3, utilizando información experimental como la obtenida de la referencia [4], la cual corresponde a los perfiles NACA 836C110 (10\% de espesor relativo) y NACA 847A216 (16\% de espesor relativo). Las Figuras 3 y 4 se presentan como ejemplo del comportamiento que tienen los coeficientes aerodinámicos con el número de Mach.

El procedimiento seguido para corregir los coeficientes aerodinámicos es el siguiente:

1.- Se busca en las gráficas el ángulo de ataque absoluto del elemento de pala y se leen los coeficientes de levantamiento y de arrastre para $M=0.3$.

2.- Para el mismo ángulo de ataque absoluto, se leen los mismos coeficientes para el número de Mach del elemento de pala, $M_{e}$.

3.- Se calculan los factores de corrección de los coeficientes de levantamiento, $F C_{l}=\frac{C_{l} \text { para } M_{e}}{C_{l} \text { para } M=0.3}$ y de arrastre, $F C_{d}=\frac{C_{d} \text { para } M_{e}}{C_{d} \text { para } M=0.3}$.

4.- Se calculan los nuevos coeficientes de levantamiento, $C_{l \text { cor. }}=C_{\text {lcalculado }} F C_{l} ; \mathrm{y}$ de arrastre $C_{d \text { cor. }}=$ $C_{d \text { calculado }} F C_{d}$ del elemento de pala.

Una vez estimados los nuevos $C_{l}$ y $C_{d}$, y empleando las ecuaciones presentadas en la sección 2.1, se recalculan las velocidades inducidas, $a$ y $b$, los diferenciales del coeficiente de tracción y del coeficiente de potencia, ambos con respecto al radio adimensional, $\frac{d C_{T}}{d r}$ y $\frac{d c_{p}}{d r}$ y finalmente el coeficiente de tracción y de potencia de la hélice, $C_{T}, C_{p}$ y el rendimiento de la hélice, $\eta$. 
Figura 3.- Variación del coeficiente de levantamiento con el número de Mach a varios ángulos de ataque del perfil NACA 847A216. Tomada de [4].

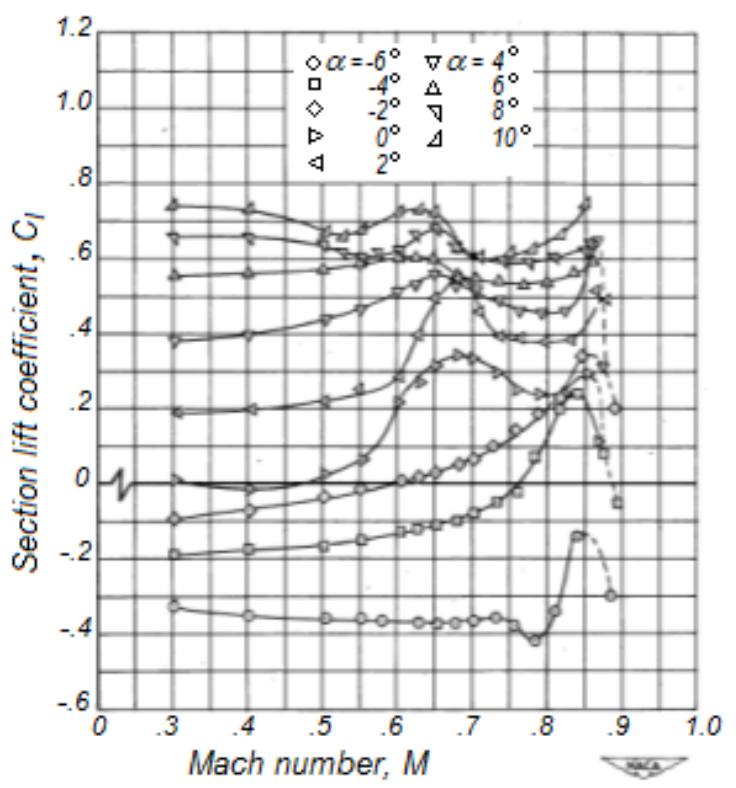

Figura 4.- Variación del coeficiente de arrastre con el número de Mach a varios ángulos de ataque del perfil NACA 847A216. Tomada de [4].

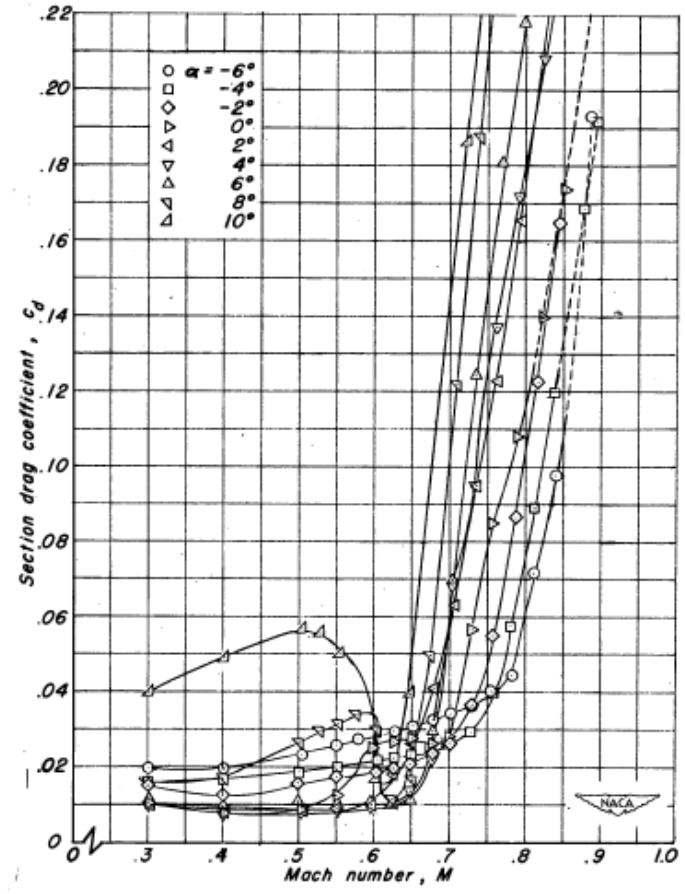

\section{RESULTADOS}

La metodología explicada en la sección 2 se aplicó a una de las hélices de la referencia [1], la hélice Navy 5868-9 de cuatro palas con perfil Clark-Y y cuya geometría se muestra en la Tabla 1, en donde también se muestran las características aerodinámicas de los elementos de pala. Las propiedades aerodinámicas fueron determinadas de la referencia [5] y corresponden a un número de Mach de 0.058 y 
a un número de Reynolds de 3200000. La Figura 1 es la correspondiente gráfica de rendimiento, $\eta$, contra la relación de avance, $J$. Para los cálculos realizados se consideró $J=2.5, J_{V s}=4.23$, un ángulo de paso geométrico, $\beta=45^{\circ}$ para la estación $r=0.75$, un número de Mach en la punta de la pala de 0.95 y un número de Mach de vuelo de 0.59.

Los resultados obtenidos se muestran en las Figuras 5 a 9. En las Figuras 5 a 8 se muestran tanto los cálculos teóricos empleando la teoría combinada como los cálculos obtenidos con la corrección por número de Mach y en la Figura 9 se muestra la variación del número de Mach a lo largo de la pala de la hélice. Los coeficientes de tracción y de potencia de la hélice se determinaron calculando el área bajo la curva correspondiente, Figuras 7 y 8 respectivamente, con la función trapz del MatLab. En la Tabla 2 se resumen los resultados obtenidos. En la ecuación (1) se utilizó un número de Mach crítico de 0.65 el cual es bastante cercano al del perfil Clark Y aunque es conveniente aclarar que el Mach crítico depende del espesor y del ángulo de ataque del elemento de pala, por lo que varía a lo largo de la pala de la hélice.

Revisando los valores de la Tabla 2, se observa que la Figura 1 proporciona un rendimiento mayor que la Ecuación (1) y que el obtenido con la corrección aquí propuesta, no así con respecto al valor obtenido con la teoría combinada. Se considera que la ecuación (1) y la corrección aquí propuesta proporcionan rendimientos más acordes a la realidad ya que consideran el efecto del número de Mach en la punta de la pala de la hélice y se considera que el valor calculado con la corrección propuesta es más cercano a la realidad ya que considera el número de Mach en todos los elementos de pala y no solo el de la punta de pala, además de considerar la variación de los coeficientes aerodinámicos de los elementos de pala con el número de Mach. Esta variación se refleja en el coeficiente de tracción el cual es bastante menor que el proporcionado por la gráfica correspondiente, ya que predomina la disminución del coeficiente de levantamiento cuando el número de Mach es mayor al número de Mach crítico. También se refleja en el incremento en el coeficiente de potencia con respecto al proporcionado por la gráfica correspondiente, ya que, para números de Mach mayores al crítico, el coeficiente de arrastre se incrementa notablemente.

Tabla 1. Características geométricas y aerodinámicas de la hélice Navy 5869-9

\begin{tabular}{|l|c|c|c|c|l|l|l|l|}
\hline $\begin{array}{l}\text { Elemento } \\
\text { de pala }\end{array}$ & $r$ & $c$ & $t / c$ & $\begin{array}{c}\beta \\
\text { grados }\end{array}$ & $\begin{array}{l}\alpha_{L=0}, \\
\text { grados }\end{array}$ & $s$ & $C_{d 0 \mathrm{~min}}$ & $\begin{array}{l}a_{0}, \\
1 / \text { grado }\end{array}$ \\
\hline 1 & 0.2000 & 0.0377 & 0.8852 & 65.9540 & -0.1589 & 0.2403 & 0.0938 & 0.0160 \\
\hline 2 & 0.2500 & 0.0450 & 0.5610 & 64.2011 & -0.3177 & 0.2289 & 0.0436 & 0.0319 \\
\hline 3 & 0.3000 & 0.0529 & 0.3600 & 61.9348 & -0.6355 & 0.2244 & 0.0231 & 0.0638 \\
\hline 4 & 0.3500 & 0.0608 & 0.2397 & 59.4796 & -1.2709 & 0.2212 & 0.0147 & 0.0914 \\
\hline 5 & 0.4000 & 0.0690 & 0.1742 & 56.8524 & -3.7142 & 0.2196 & 0.0113 & 0.0985 \\
\hline 6 & 0.4500 & 0.0743 & 0.1357 & 54.2292 & -3.9094 & 0.2102 & 0.0097 & 0.1011 \\
\hline 7 & 0.5000 & 0.0764 & 0.1174 & 51.8963 & -3.8811 & 0.1947 & 0.0091 & 0.1021 \\
\hline 8 & 0.5500 & 0.0755 & 0.1078 & 49.9287 & -3.8035 & 0.1747 & 0.0088 & 0.1026 \\
\hline
\end{tabular}




\begin{tabular}{|l|l|l|l|l|l|l|l|l|}
\hline 9 & 0.6000 & 0.0731 & 0.1011 & 48.3841 & -3.7287 & 0.1551 & 0.0086 & 0.1029 \\
\hline 10 & 0.6500 & 0.0700 & 0.0963 & 47.0551 & -3.6702 & 0.1370 & 0.0084 & 0.1031 \\
\hline 11 & 0.7000 & 0.0661 & 0.0924 & 45.9006 & -3.6240 & 0.1202 & 0.0083 & 0.1033 \\
\hline 12 & 0.7500 & 0.0613 & 0.0886 & 45.0000 & -3.5818 & 0.1041 & 0.0082 & 0.1035 \\
\hline 13 & 0.8000 & 0.0563 & 0.0866 & 44.1010 & -3.5634 & 0.0895 & 0.0082 & 0.1036 \\
\hline 14 & 0.8500 & 0.0507 & 0.0837 & 43.3019 & -3.5407 & 0.0760 & 0.0081 & 0.1037 \\
\hline 15 & 0.9000 & 0.0445 & 0.0828 & 42.6812 & -3.5348 & 0.0629 & 0.0081 & 0.1037 \\
\hline 16 & 0.9500 & 0.0375 & 0.0818 & 42.1233 & -3.5298 & 0.0503 & 0.0080 & 0.1038 \\
\hline
\end{tabular}

Fuente: elaboración propia.

Tabla 2. Resultados obtenidos.

\begin{tabular}{|l|l|l|l|}
\hline Método & $\mathrm{Ct}$ & $\mathrm{Cp}$ & $\eta$ \\
\hline Ecuación (1) & & & 0.12 \\
\hline Gráficas [1] & 0.0275 & 0.1200 & 0.57 \\
\hline Teoría combinada & 0.0198 & 0.0705 & 0.70 \\
\hline Corrección & 0.0039 & 0.0975 & 0.10 \\
\hline
\end{tabular}

Fuente: elaboración propia.

Figura 5. Variación del $C_{d}$ a lo largo de la pala.

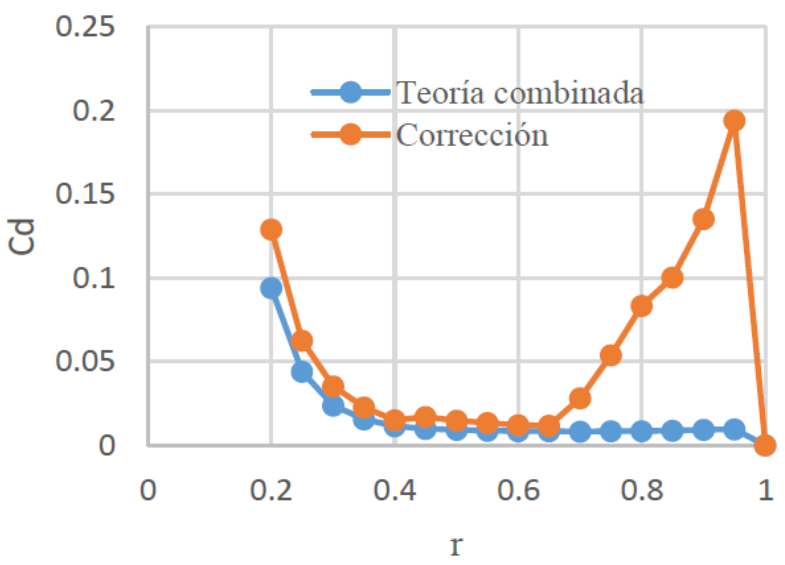

Fuente: elaboración propia.

Figura 6. Variación del $C_{l}$ a lo largo de la pala.

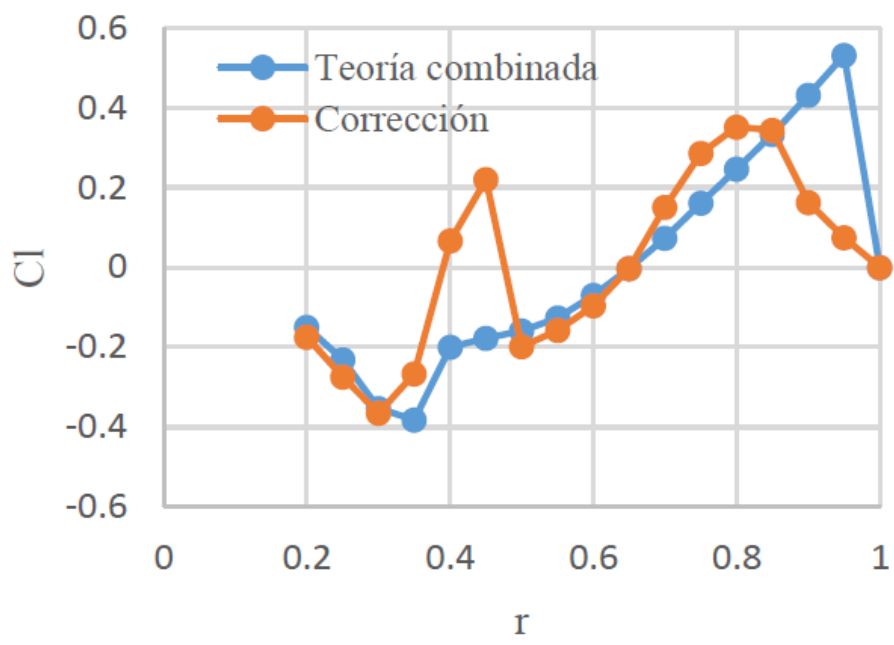

Fuente: elaboración propia. 


\section{CONCLUSIONES}

Figura 7. Variación del $C_{T}$ a lo largo de la pala.

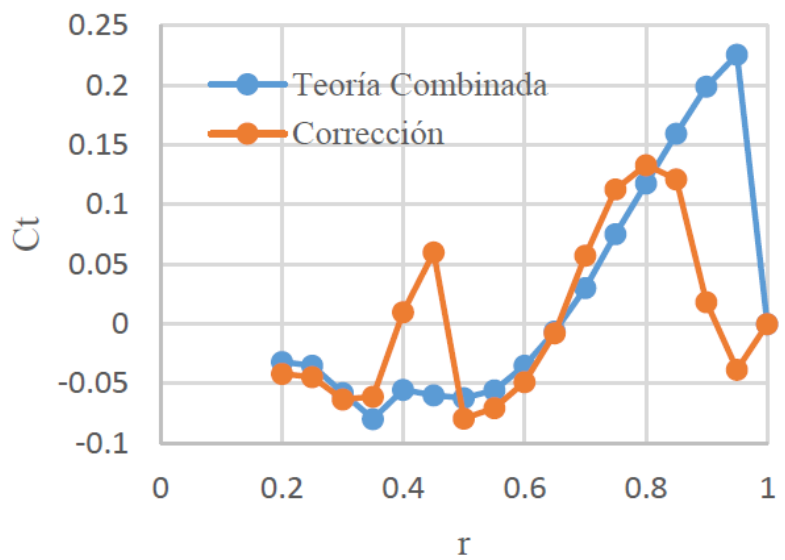

Fuente: elaboración propia.

Figura 8. Variación del $C_{p}$ a lo largo de la pala.

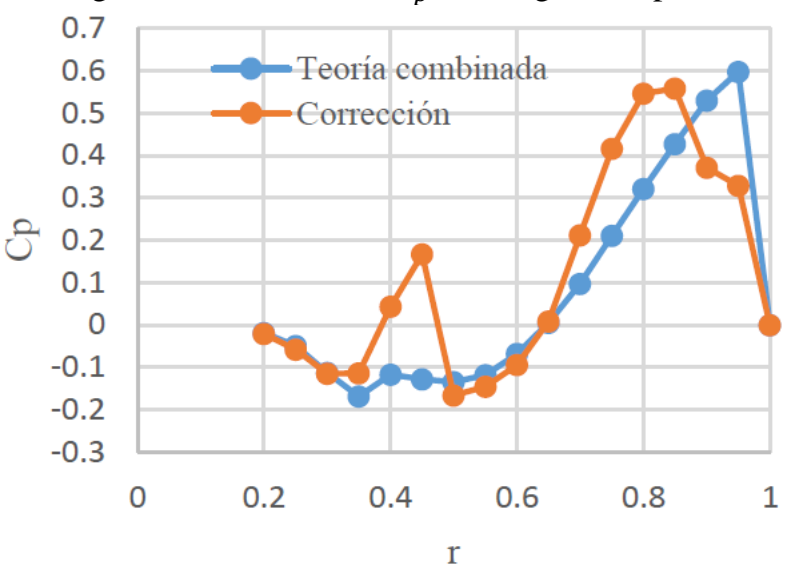

Fuente: elaboración propia.

Figura 9. Variación del número de Mach a lo largo de la pala.

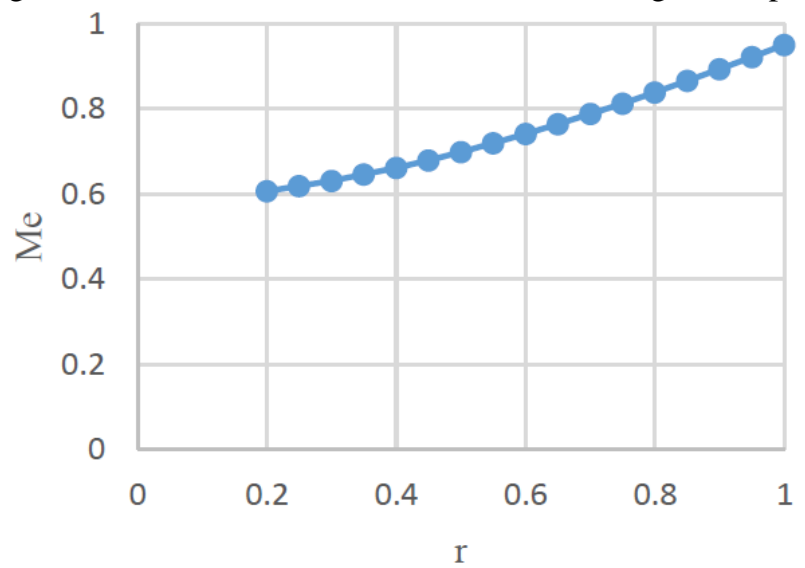

Fuente: elaboración propia. 


\section{REFERENCIAS}

[1] E. P. Hartman, D. Biermann, The Aerodynamic Characteristics of Full-Scale Propellers Having 2, 3, and 4 Blades of Clark Y and R. A. F. 6 Airfoil Sections, Report No. 640, National Advisory Committee for Aeronautics, Washington, D. C., 1938.

[2] D. R. Greatrix, Powered Flight, The Engineering of Aerospace Propulsion, (eBook), DOI 10.1007/978-1-4471-2485-6, Springer-Verlag, London, 2012.

[3] Richard von Misses, Theory of Flight, Dover Publications, Inc., New York, 1959.

[4] Donald J. Graham, The Development of Cambered Airfoil Sections Having Favorable Lift Characteristics at Supercritical Mach Numbers, NACA Technical Note No. 1771, Washington, 1948.

[5] E. N. Jacobs, K. E. Ward, Robert M. Pinkerton, The Characteristics of 78 Related Airfoil Sections From Tests in the Variable-Density Wind Tunnel, NACA Report No. 460, Langley Memorial Aeronautical Laboratory, 1933. 